\title{
Establishing a Pediatric Acute-Onset Neuropsychiatric Syndrome Clinic: Baseline Clinical Features of the Pediatric Acute-Onset Neuropsychiatric Syndrome Cohort at Karolinska Institutet
}

\author{
Caroline Gromark, MD, ${ }^{1-3}$ Robert A. Harris, PhD, ${ }^{1}$ Ronny Wickström, MD, PhD, ${ }^{4,5}$ \\ AnnaCarin Horne, MD, PhD, ${ }^{4,6}$ Maria Silverberg-Mörse, MD, ${ }^{2}$ \\ Eva Serlachius, MD, $\mathrm{PhD}{ }^{1,3}$ and David Mataix-Cols, $\mathrm{PhD}^{1-3}$
}

\begin{abstract}
Objectives: Pediatric acute-onset neuropsychiatric syndrome (PANS) is a descriptive clinical entity defined by the abrupt onset of psychiatric and somatic symptoms leading to significant loss of function. Data on well-characterized PANS patients are limited, biomarkers have yet to be identified, and a solid evidence base to guide treatment is lacking. In this study, we present our experience of a systematic evaluation of the first 45 patients included in a Swedish cohort.

Methods: During the period 2014-2018, our clinic received 100 referrals regarding suspected PANS. All patients underwent a standardized psychiatric/medical evaluation by a child/adolescent psychiatrist and a clinical psychologist or a nurse. Those with severe symptoms were also assessed by a pediatric neurologist and a pediatric rheumatologist. Laboratory tests were obtained at different time points in an attempt to capture an active disease state.

Results: Of the 100 referrals, 45 met strict PANS criteria and consented to participate in a long-term follow-up study. The median age at intake was 7.2 years (range 3.0-13.1) and 56\% were male. Ninety-three percent fulfilled both criteria for acute/atypical onset of PANS symptoms and having had an infection in relation to onset. Sixteen percent had an onset of an autoimmune or inflammatory disorder in temporal relation to the onset of PANS-related symptoms. The most common onset symptoms were obsessive-compulsive disorder (89\%), anxiety (78\%), and emotional lability (71\%). Twenty-four percent had a preexisting autoimmune disease (AD) and $18 \%$ a preexisting psychiatric/neuropsychiatric diagnosis. Sixty-four percent of biological relatives had at least one psychiatric disorder and $76 \%$ at least one $\mathrm{AD}$ or inflammatory disorder. Complement activation (37\%), leukopenia (20\%), positive antinuclear antibodies (17\%), and elevated thyroid antibodies (11\%) were the most common laboratory findings.

Conclusions: In our PANS cohort, there was a strong indication of an association with AD. Further work is needed to establish whether any of the potential biomarkers identified will be clinically useful. Long-term follow-up of these patients using the Swedish national registers will enable a deeper understanding of the course of this patient group.
\end{abstract}

Keywords: PANS, PANDAS, OCD, tics, autoimmune disease

${ }^{1}$ Department of Clinical Neuroscience, Karolinska Institutet, Stockholm, Sweden.

${ }^{2}$ BUP OCD and Related Disorders, Stockholm Health Care Services, Stockholm County Council, Stockholm, Sweden.

${ }^{3}$ Centre for Psychiatry Research, Stockholm Health Care Services, Stockholm County Council, Stockholm, Sweden.

${ }^{4}$ Department of Women's and Children's Health, Karolinska Institutet, Stockholm, Sweden.

Divisions of ${ }^{5}$ Pediatric Neurology and ${ }^{6}$ Pediatric Rheumatology, Karolinska University Hospital, Solna, Sweden.

Funding: The research was funded by Stockholm County Council, Jane and Dan foundation, Fonden för Rehabilitering och Medicinsk Forskning (FRF-stiftelsen), and Psykiatrifonden.

(c) Caroline Gromark et al. 2019; Published by Mary Ann Liebert, Inc. This Open Access article is distributed under the terms of the Creative Commons License (http://creativecommons.org/licenses/by/4.0), which permits unrestricted use, distribution, and reproduction in any medium, provided the original work is properly cited. 


\section{Introduction}

$\mathbf{T}$ HERE IS INCREASING EVIDENCE for an association between autoimmune disease (AD) and neuropsychiatric disorders (Najjar et al. 2013; Mataix-Cols et al. 2018). Pediatric acute-onset neuropsychiatric syndrome (PANS) is a descriptive entity of disputed validity and for which there are currently no defined biomarkers (Chang et al. 2015; Hesselmark and Bejerot 2017a, 2017 b). It affects young children, often in temporal relation to an uncomplicated infection, resulting in abrupt onset of obsessivecompulsive disorder (OCD) and/or anorexia, emotional lability, and a wide range of somatic symptoms (Swedo et al. 1998; Chang et al. 2015; Hesselmark and Bejerot 2017a, 2017b). The severity of symptoms may lead to significant loss of function, impacting both the children themselves and their families (Frankovich et al. 2015). Other diseases of known etiology, but with a similar clinical picture, such as Sydenham's chorea, systemic lupus erythematosus, or other inflammatory encephalitides, such as anti-NMDA receptor encephalitis, need to be excluded (Dalmau et al. 2008; Dale and Brilot 2012; Hacohen et al. 2013; Ramanathan 2014). While the etiology is unknown, infectious agents such as group A streptococci (GAS), mycoplasma, and Epstein-Barr virus as well as AD and inflammatory disorders have been described as contributing to the pathogenic mechanisms and potential triggers for the constellation of symptoms that constitute PANS (Kurlan et al. 2008; Leckman et al. 2011; Brimberg et al. 2012; Cutforth et al. 2016; Mahony et al. 2017a, 2017b).

Based on clinical experience and previous research, the PANS Research Consortium (Cooperstock et al. 2017; Frankovich et al. 2017; Thienemann et al. 2017) has developed expert consensus guidelines for psychiatric, infectious, and immunomodulatory treatments, respectively, but the lack of well-defined cohorts of patients, absence of reliable biomarkers, and lack of conclusive clinical trials have complicated the interpretation of results. Clinical data of well-characterized PANS patients are still limited and more evidence is needed to support the current treatment routines that have been developed mainly according to clinical experience, case reports, and case series. Previous research has suggested that immunomodulatory and/or anti-inflammatory treatments may be beneficial in some cases, but evidence is inconclusive (Latimer et al. 2015; Farhood et al. 2016; Williams et al. 2016; Brown et al. 2017a, 2017b; Spartz et al. 2017; Sigra et al. 2018). Definitive clinical trials are sorely needed to guide clinical decision-making.

Our specialist OCD and related disorders clinic started accepting potential PANS referrals in November 2014, and since then, we have gathered a well-defined cohort of patients fulfilling criteria for PANS, together with a large amount of longitudinal clinical and biological data. We herein describe development of the PANS clinic and preliminary clinical and laboratory data for the first 45 patients included in the cohort. This research is part of a larger project that will gather long-term follow-up data from these patients through linkage with the Swedish population-based registers, with the aim of identifying potential clinical and biological predictors of clinical course for this group.

\section{Methods}

\section{Clinical setting}

All study participants were recruited from a specialist pediatric OCD and related disorders outpatient clinic in Stockholm, Sweden. The clinic primarily receives referrals from Child and Adolescent Psychiatry Services (CAMHS) and pediatric services across the entire Stockholm region and occasionally from other Swedish regions and Nordic countries. All patients and their parents/legal guardians routinely fill in questionnaires before their first appointment with the multidisciplinary clinical team comprising child/adolescent psychiatrists, clinical psychologists, and nurses. This information is then used to conduct a more focused and efficient, face-to-face diagnostic assessment. During the first 3-hour appointment, detailed sociodemographic and clinical information is gathered from patients and their parents and clinical diagnoses are made according to International Classification of Diseases, 10th Revision (ICD-10), and Diagnostic and Statistical Manual of Mental Disorders, 5th edition (DSM-5), criteria (World Health Organization 2011; American Psychiatric Association 2013). After this assessment, patients are either offered treatment at the clinic or referred to more appropriate services. For all patients undertaking treatment at the clinic, assessments are repeated at post-treatment and at several fixed follow-up times: 3, 6, and 12 months after the end of the treatment. All patients assessed at the clinic are routinely asked to participate in research studies, including a long-term follow-up project with aims to evaluate the broad long-term outcomes of our patients with the help of the Swedish populationbased registers.

In 2014, the clinic started accepting referrals of potential PANS cases and, as the demand increased, we established a PANS team within our clinic, currently consisting of a child and adolescent psychiatrist, a nurse, and two clinical psychologists. The PANS team closely collaborates with the pediatric neuroinflammation team at the Karolinska University Hospital, which creates a multispecialist environment with child and adolescent psychiatry, pediatric rheumatology, and pediatric neurology. The collaboration has enabled development of Sweden's first clinical routines for evaluation and management of youths with PANS in consensus with pediatric neurology, pediatric rheumatology, and CAMHS across Stockholm in April 2018. These clinical routines resemble, but are not identical to, other guidelines recently reported in the United States (Cooperstock et al. 2017; Frankovich et al. 2017; Thienemann et al. 2017). Verified infections are treated with antibiotics, but because clinical trials are still inconclusive regarding the benefits of long-term antibiotics, the Stockholm clinical routines discourage their prophylactic use until firmer evidence becomes available. The treatment routines also include a requirement for neurological clinical signs-electroencephalography (EEG) and/or magnetic resonance imaging (MRI) abnormalities and/or biomarkers (in blood and/or cerebrospinal fluid [CSF]) — that suggest an active neuroinflammation before intravenous immunoglobulin (IVIG) treatment is considered.

All young people and their parents gave written consent to participate in the current study, which was approved by the Regional Ethics Review Board in Stockholm (reference number EPN 2015/1977-31/4).

\section{Clinical evaluations}

All suspected PANS cases underwent a thorough psychiatric and medical evaluation at first presentation at the clinic. A child and adolescent psychiatrist, a clinical psychologist, and a specialist psychiatric nurse carried out the assessments. The psychiatric evaluation included a full developmental and psychiatric history as well as relevant validated rating scales depending on primary symptoms (such as the Children's Yale-Brown Obsessive Compulsive Scale [CYBOCS] for OCD or the Yale Global Tic Severity Scale [YGTSS] for tics) (Goodman et al. 1989; Leckman et al. 
1989; Storch et al. 2010; McGuire et al. 2018). A clinician assessed global psychiatric symptom severity and improvement at each visit with the Clinical Global Impressions-Severity Scale (CGI-S) and the Clinical Global Impressions-Improvement Scale (CGI-I), respectively. In this study, CGI-S and CGI-I were employed as measures of general psychopathology, rather than measures of specific forms of psychopathology. Global functioning was assessed at each visit using the Children's Global Assessment Scale (CGAS) (Shaffer et al. 1983; Busner and Targum 2007). As the study progressed, it became apparent that time-consuming instruments such as the CYBOCS and YGTSS were difficult to administer to our youngest patients (as young as 3 or 4 in our cohort), some of whom had difficulties communicating their symptoms. Parents are often unaware of the child's obsessions and can only report on observable behavior. Finally, PANS patients often present with a wide range of complaints, sometimes OCD being the least prominent. For these reasons, we chose not to use the CYBOCS as part of our routine baseline assessment, unless OCD was the main clinical presentation and the patient was old and/or communicative enough to be accurately assessed.

The medical evaluation included developmental and medical history, vaccination records, a thorough somatic assessment focusing on signs of infection, and rheumatological and neurological signs. Depending on severity of symptoms and clinical signs, further assessments such as EEG, MRI, or lumbar puncture for CSF were arranged through pediatric neurology and/or rheumatology clinics. Family history was carefully recorded in a systematic way using a comprehensive checklist of psychiatric, neurological, and autoimmune/inflammatory disorders in first-, second-, and thirddegree relatives.

Neuropsychological testing was performed if the patient showed signs of cognitive impairment, if the disease had a deteriorating course, and/or to evaluate treatment outcomes. Medical records, including those from primary care physicians, emergency care visits, and inpatient units, were reviewed by the treating clinician responsible for the PANS team (C.G.).

\section{Laboratory workup}

The team developed a laboratory protocol that was used for every patient. The protocol included basic blood measurements of C-reactive protein (CRP), erythrocyte sedimentation rate, hemoglobin, and complete blood count (CBC), indicators for liver and kidney function, thyroid tests, celiac test, and rheumatological markers such as antinuclear antibodies (ANAs), histone antibodies, protein fractions, immunoglobulins, complements, and serum amyloid A (SAA), as well as some of the more frequently tested cytokines (interleukin [IL]-1- $\beta$, IL-6, IL-8, and tumor necrosis factor $-\alpha[\mathrm{TNF}-\alpha])$. The reference values used were those used clinically by the Karolinska laboratory (Bäck et al. 1999, Roche Diagnostics GmbH 2009, Yamada 1999). Blood tests were ordered at the time of first assessment at the clinic and at some intervals during follow-up, more frequently if clinically necessary because of a more severe disease trajectory. When the patient exhibited signs of infection, cultures were taken. The laboratory workup evolved slightly over the course of the study period, following the clinical development of PANS care. Some tests, such as measurement of histone antibodies, cytokines, and SAA, have therefore not been conducted for all patients.

The laboratory results reported in this article represent findings at one point in time during onset, flare (defined as CGI-S equal to or higher than 4 and marked as a worsening period in patient records), or during a chronic-static or progressive course. If the full laboratory workup was made repeatedly for the same patient, the point with the highest CGI-S was chosen. In the few cases where CGI-S was not sufficient to differentiate the severity of flares, the full symptom description of the flare as described in patient records was assessed. We therefore believe that the findings represent, as far as possible, an active disease state. Some patients showed a positive ANA, complement activation, and/or elevated SAA at another point in the disease course than the one chosen, for example, during a less severe flare. These findings have not been included in the table. Patients with diagnosed celiac disease, eating gluten-free and therefore having no detectable transglutaminase antibodies, have been included as being transglutaminase antibody negative if the laboratory test at the time point was negative. Streptococcal cultures were only included if taken at the same time point as the other laboratory tests.

\section{Statistical analyses}

Statistical analysis was conducted using STATA software (version STATA/IC15.1 for Mac). Analyses were largely descriptive in nature. When comparing subgroups of patients within the cohort, t-tests for continuous variables and chi-square tests for proportions were employed. A $p$-value below 0.05 was considered statistically significant.

\section{Results}

\section{Referral flow}

Between November 2014 and March 2018, our clinic received 100 referrals regarding suspected PANS. Forty-seven patients met the criteria for PANS, even though it was difficult to assess the acuteness of onset in some cases. Of these, 45 consented to participate in our long-term follow-up study and future linkage to the Swedish registers (Fig. 1). The most common reasons for exclusion were absence of core psychiatric symptoms such as OCD and/or anorexia, insidious symptom onset, absence of somatic signs, an$\mathrm{d} /$ or spontaneous resolution of symptoms before the assessment took place.

\section{Demographic and clinical characteristics}

The sociodemographic and clinical characteristics of the cohort are presented in Table 1. Fifty-six percent of patients were male. The mean age at symptom onset was 7.5 years (standard deviation [SD] 2.5, range 3.0-13.1, median 7.2 years) and the mean age at presentation at the clinic/first assessment was 9.0 years (SD 3.1, range 3.3-15.7, median 9.0 years). Developmental abnormalities were noted in patient records in $18 \%$ of the cohort, the same fraction as having had a preexisting psychiatric and/or neuropsychiatric diagnosis. A slightly higher fraction, 24\%, had a preexisting disease or disorder commonly considered to have an autoimmune or inflammatory etiology, the most common being severe asthma, severe atopic eczema, severe and multiple nutritional allergies, celiac disease, postinfectious arthritis, and HenochSchönlein's purpura.

Almost all patients (93\%) clearly fulfilled both criteria for acute onset ( $<72$ hours) of PANS-related symptoms and having had an infection in temporal relation to symptom onset. For the remaining $7 \%$ of cases, it was not possible to fully confirm the type of onset because of a long time having passed between onset and presentation at the clinic or insufficiently detailed patient records, but the parent-reported clinical history was considered distinct enough for 


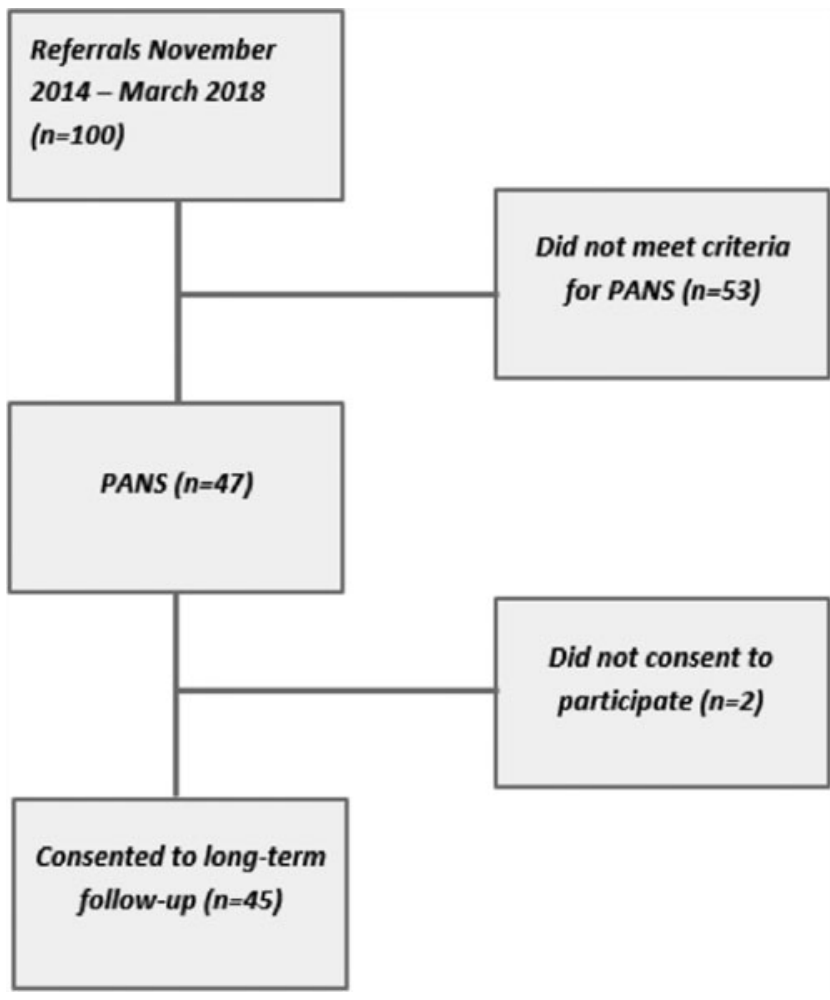

FIG. 1. Flow chart of study participants. PANS: individuals fulfilling PANS criteria. Did not meet criteria for PANS: individuals not fulfilling PANS criteria due to absence of core symptoms such as obsessive-compulsive disorder and/or anorexia, insidious symptom onset, lack of somatic signs, or spontaneous resolution of symptoms before the assessment. PANS, pediatric acute-onset neuropsychiatric syndrome.

the patient to be included in the cohort. Sixteen percent had an onset of an autoimmune or inflammatory disorder in temporal relation to the onset of PANS-related symptoms. Among these disorders, autoimmune thyroiditis, celiac disease, and diabetes mellitus type 1 were represented.

\section{Onset symptoms}

The main onset symptoms are presented in Figure 2. In the cohort, $89 \%$ were assessed to fulfill criteria for OCD at the time of onset. Anxiety (including separation anxiety) was evident in $78 \%$ and emotional lability and/or depression in $71 \%$. Sleep disorders, including insomnia, sleep disturbances, and frequent nightmares, were reported by $69 \%$ of patients. Sixty-two percent had complex tics, which were often both motor and vocal, resulting in pain or loss of function. Other forms of motor abnormalities (including chorea and choreiform movements, dystonia, perception of muscle weakness, and difficulties with gross motor control as well as fine motor skills) were present in $60 \%$ of patients. Attention deficit was reported by $63 \%$ and hyperactivity by $43 \%$ of patients. Despite the young age of the cohort, $50 \%$ reported deterioration in school performance since symptom onset. Irritability/aggression and regressive behaviors were reported in $44 \%$ and $40 \%$, respectively. Half of the patients reported sensory abnormalities, including hypersensitivity to touch/clothes, sound, and light. Forty percent had an eating disorder (significant loss of appetite resulting in weight loss, avoidant-restrictive food intake disor-
Table 1. Patient Characteristics at Intake $(N=45)$

\begin{tabular}{lc}
\hline Patient demographics & Frequencies/means (SD) \\
\hline Male & $25 / 45(56 \%)$ \\
Mean age at symptom onset (years) & 7.5 (SD 2.5) \\
Mean age at intake (years) & 9.0 (SD 3.1) \\
$\begin{array}{l}\text { Developmental abnormalities } \\
\quad \text { psychomotor, language disorder, }\end{array}$ & $8 / 45(18 \%)$ \\
$\quad$ and/or learning disability) & \\
Preexisting & $8 / 45(18 \%)$ \\
$\quad$ psychiatric/neuropsychiatric & \\
$\quad$ diagnoses & \\
Preexisting autoimmune disease & $11 / 45(24 \%)$ \\
$\quad$ or inflammatory disorder & \\
CGAS at intake & \\
CGI-S at intake & $30($ SD 10.1) \\
Acute symptom onset & 3.8 (SD 0.9) \\
Infection in temporal relation & $42 / 45(93 \%)$ \\
$\quad$ to symptom onset & $42 / 45(93 \%)$ \\
Onset of autoimmune disease & \\
$\quad$ or inflammatory disorder in & \\
$\quad$ temporal relation to symptom onset &
\end{tabular}

${ }^{a}$ Available for 43 patients only.

CGAS, Children's Global Assessment Scale; CGI-S, Clinical Global Impressions-Severity Scale; SD, standard deviation.

der, or other OCD-related eating disorder). Somatic symptoms such as urinary problems and pain were reported in $44 \%$ and $38 \%$, respectively.

The mean CGAS and CGI-S values at presentation were 50 (SD 10.1, range 21-70) and 3.8 (SD 0.9, range 2-6), respectively. The wide range in CGAS rating indicates that some patients were severely ill at onset, while others were mildly ill, but still fulfilled PANS criteria (Table 1).

\section{Somatic signs}

Somatic signs at onset/presentation are presented in Table 2. Slightly fewer than half of the patients, $46 \%$, exhibited ear, nose, and throat (ENT) infection signs at onset/first assessment. Nine percent had palatal petechiae as a suspected sign of streptococcal infection (Mahony et al. 2017b). Skin abnormalities, including dermatographia, livedo, rashes, and/or eczema, were present in $61 \%$ of patients. From the somatic assessment, $23 \%$ had some kind of neurological abnormality (the most common being ataxia or side differences in force or reflexes of the extremities), $23 \%$ had motor abnormalities, and $23 \%$ exhibited choreiform movements. Choreatic movements at the time of first assessment were only evident in one patient $(1 / 41 ; 2 \%)$. Only one patient $(1 / 28 ; 4 \%)$ presented with a heart abnormality upon auscultation. It consisted of a heart murmur, which after ultrasound assessment, was considered physiological and not due to inflammatory changes.

\section{Laboratory findings}

Laboratory findings are presented in Table 3 . The most common CBC abnormality was mild leukopenia $(9 / 45 ; 20 \%)$. Ferritin deficiency and vitamin D deficiency were frequently noted in the cohort, but are both relatively frequent in the normal pediatric population (Parkin et al. 2017; Yesiltepe and Hatun 2018). In the total cohort, $11 \%$ had elevated thyroperoxidase antibodies (antiTPO) and 5\% had elevated transglutaminase antibodies, indicating thyroid and celiac disease, respectively. A full $37 \%$ had complement 


\section{SYMPTOMS ONSET/PRESENTATION}

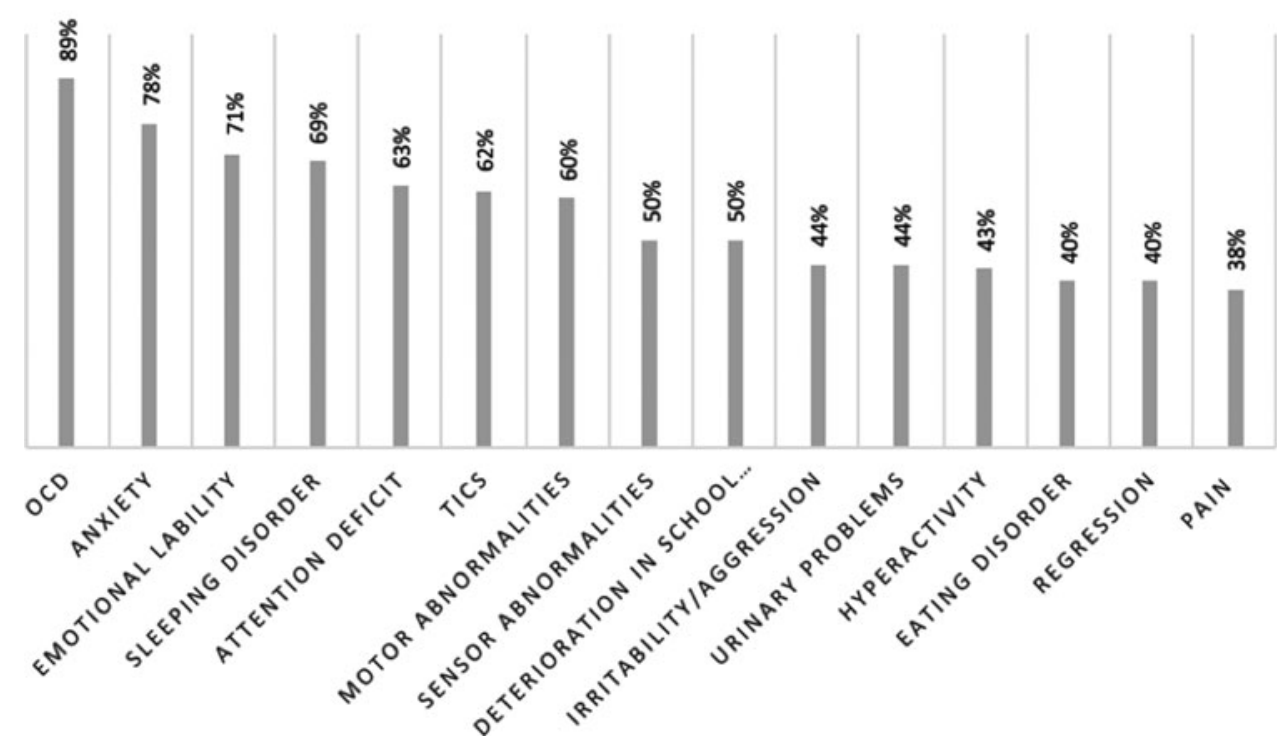

FIG. 2. Onset symptoms as described in patient records and/or at presentation at the clinic $(n=45)$. Values do not add up to $100 \%$ since most patients had more than one symptom at onset. OCD, obsessive-compulsive disorder.

deficiencies indicating complement activation. Seventeen percent had positive ANAs and $14 \%$ had an elevated SAA. Eleven percent had low immunoglobulin $\mathrm{G}$ (IgG) and 9\% had low immunoglobulin A (IgA). None of the patients had low immunoglobulin M (IgM). None of the patients had a clear increase in any of the serum cytokines tested for (IL-1- $\beta$, IL-6, IL-8, and TNF- $\alpha$ ). Streptococcal cultures were rarely taken at the same time as the blood tests, but when performed were positive for GAS in $50 \%$ of the 10 tested cases.

\section{Family history of psychiatric, autoimmune, and inflammatory disorders}

Data of family history of psychiatric and AD/inflammatory disorders are presented in Table 4, which combines data for first-, second-, and third-degree relatives. In all the relatives combined, $64 \%$ had at least one psychiatric disorder, the most common being depression (36\%), attention-deficit/hyperactivity disorder (20\%), OCD (16\%), anxiety $(16 \%)$, tics $(13 \%)$, and autism spectrum disorder $(11 \%)$.

\section{Table 2. Somatic Findings at First Clinical Evaluation}

\begin{tabular}{lc}
\hline Somatic sign onset/presentation & Frequencies $^{\mathrm{a}}$ \\
\hline Heart abnormalities & $1 / 28(4 \%)$ \\
Lung abnormalities & $0 / 27(0 \%)$ \\
Stomach abnormalities & $0 / 13(0 \%)$ \\
Joint abnormalities & $0 / 16(0 \%)$ \\
ENT infection signs & $16 / 35(46 \%)$ \\
Palatal petechiae & $2 / 22(9 \%)$ \\
Skin abnormalities (dermatographia, livedo, severe & $11 / 18(61 \%)$ \\
$\quad$ dryness, redness, rash, and eczema, etc.) & \\
Neurological abnormalities & $7 / 31(23 \%)$ \\
Abnormalities in motor function & $7 / 30(23 \%)$ \\
Chorea & $1 / 41(2 \%)$ \\
Choreiform movements & $6 / 26(23 \%)$
\end{tabular}

${ }^{\mathrm{a}}$ These signs were only assessed in a subsample of participants. ENT, ear, nose, and throat.
Table 3. Laboratory Findings Corresponding to One Point in Time During Symptom Onset, Flare (Defined as Clinical Global Impressions-Severity Scale Equal to or Higher Than 4 and Marked as a Worsening Period in Patient Records), or During a Chronic-Static or Progressive Course, Compared with POPUlation NoRMS

\begin{tabular}{lc}
\hline Laboratory findings & Frequencies $^{\mathrm{a}}$ \\
\hline CBC abnormalities & $26 / 45(58 \%)$ \\
Elevated ESR & $6 / 43(14 \%)$ \\
Elevated CRP & $4 / 37(11 \%)$ \\
Low ferritin & $8 / 39(21 \%)$ \\
Low vitamin D & $10 / 36(28 \%)$ \\
TSH abnormalities & $4 / 40(10 \%)$ \\
Low T4 & $0 / 40(0 \%)$ \\
Anti-TPO & $4 / 38(11 \%)$ \\
Transglutaminase antibodies & $2 / 40(5 \%)$ \\
ANAs & $7 / 41(17 \%)$ \\
Histone antibodies & $0 / 31(0 \%)$ \\
Elevated SAA & $3 / 22(14 \%)$ \\
Complement activation & $13 / 35(37 \%)$ \\
Low IgG & $4 / 36(11 \%)$ \\
Low IgA & $3 / 34(9 \%)$ \\
Low IgM & $0 / 34(0 \%)$ \\
Elevated IL-1- $\beta$ & $0 / 25(0 \%)$ \\
Elevated IL-6 & $1 / 25(4 \%)$ \\
Elevated IL-8 & $0 / 25(0 \%)$ \\
Elevated TNF- $\alpha$ & $0 / 24(0 \%)$ \\
Positive strep/throat culture & $5 / 10(50 \%)$
\end{tabular}

${ }^{\text {a }}$ These tests were only assessed in a subsample of participants.

ANAs, antinuclear antibodies; CBC, complete blood count; CRP, Creactive protein; ESR, erythrocyte sedimentation rate; IgA, immunoglobulin A; IgG, immunoglobulin $\mathrm{G}$; IgM, immunoglobulin $\mathrm{M}$; IL, interleukin; SAA, serum amyloid A; TNF- $\alpha$, tumor necrosis factor- $\alpha$; TPO, thyroperoxidase; TSH, thyroid-stimulating hormone. 
Table 4. Family History of Psychiatric Disorders and Autoimmune Diseases in First-, Second-, and Third-Degree Relatives of Study PARTICIPANTS $(N=45)$

\begin{tabular}{lr}
\hline Family history & Frequencies \\
\hline Psychiatric/neuropsychiatric disorder in first-, second-, and third- \\
degree relatives \\
Attention-deficit/hyperactivity disorder & $9 / 45(20 \%)$ \\
Autism spectrum disorder & $5 / 45(11 \%)$ \\
Tics & $6 / 45(13 \%)$ \\
Obsessive-compulsive disorder & $7 / 45(16 \%)$ \\
Anxiety disorders & $7 / 45(16 \%)$ \\
Depression & $16 / 45(36 \%)$ \\
Any & $29 / 45(64 \%)$ \\
Autoimmune disease/inflammatory disorder in first-, & second-, and \\
third-degree relatives & \\
Thyroid disease & $12 / 45(27 \%)$ \\
Celiac disease & $2 / 45(4 \%)$ \\
SLE & $0 / 45(0 \%)$ \\
Rheumatoid arthritis/juvenile idiopathic arthritis & $11 / 45(24 \%)$ \\
Psoriasis & $7 / 45(16 \%)$ \\
Diabetes mellitus type 1 & $2 / 45(4 \%)$ \\
Inflammatory bowel disease & $3 / 45(7 \%)$ \\
Neuroinflammatory disease (multiple sclerosis & $4 / 45(9 \%)$ \\
and amyotrophic lateral sclerosis) & \\
Other (vitiligo, IgA nephritis, vasculitis, & $20 / 45(44 \%)$ \\
polymyalgia rheumatica, or rheumatic fever) & \\
Any & $34 / 45(76 \%)$ \\
\hline
\end{tabular}

SLE, systemic lupus erythematosus.

Family history of AD or inflammatory disorder was even more common, with $76 \%$ of all relatives having had at least one AD or inflammatory disorder. The most frequent were thyroid disease (27\%), rheumatoid arthritis/juvenile idiopathic arthritis (24\%), and psoriasis $(16 \%)$. In total, $76 \%$ of individuals in the cohort had a relative with at least one $\mathrm{AD}$ and/or inflammatory disorder (Table 4).

\section{Post hoc analyses}

Given the high rates of $\mathrm{AD}$ in the cohort and their relatives, we conducted unplanned analyses comparing an autoimmune group (either the patient or a first-degree relative had at least one autoimmune disorder; $n=26$ ) and a nonautoimmune group (neither the patient nor the first-degree relative had an autoimmune disorder; $n=19)$. No statistically significant differences emerged between these groups regarding any of the sociodemographic, clinical, or laboratory variables (Supplementary Tables S1-S5).

\section{Discussion}

PANS is a relatively new descriptive entity of unclear validity and still uncertain clinical utility. There is a great need for better characterization and long-term follow-up of this patient group to identify clinical and biological markers to guide treatment development. The PANS entity describes a wide range of symptoms, presenting a clinical challenge both when trying to assess symptoms and to evaluate treatments. In our experience, global functioning scales, such as CGI and CGAS, were easier to administer than syndrome-specific instruments such as the CYBOCS.

All patients included in the cohort fulfilled PANS criteria according to patient records, psychiatric and medical histories, and the clinical evaluation. In some cases, the time between symptom onset and presentation at the clinic/first assessment was several years (range 0.04-10.8) and the time of symptom onset was therefore more difficult to accurately assess. Compared with previously reported data from the Stanford cohort (Frankovich et al. 2015 ), the patients included in our cohort were $\sim 2$ years younger at onset (7.5 vs. 9.6 years), but had almost the same delay before presenting at the clinic (presenting at 9.0 vs. 11.8 years). We observed a more even distribution between males and females $(56 \%$ males in our cohort vs. $77 \%$ males in the Stanford cohort).

PANS is sometimes described as a disorder striking previously healthy children. This is not the clinical picture we observe in our cohort as almost a fifth of the patients $(18 \%)$ had a previous psychiatric or neuropsychiatric diagnosis. In these patients, the PANS onset led to a sudden and severe worsening of psychiatric symptoms as well as development of a wider symptom spectrum. Interestingly, almost a quarter of the patients $(24 \%)$ had a previous $\mathrm{AD}$ or inflammatory disorder and as many as $16 \%$ had an onset of an $\mathrm{AD}$ or an inflammatory disorder in temporal relation to the PANS onset.

We noted that somatic symptoms, such as enuresis, pain, and skin rashes, were less often reported if not asked or looked for specifically. Even though the somatic examination revealed relatively few abnormalities in most patients, it needs to be performed systematically to detect more severe somatic signs that would otherwise go unnoticed. This demonstrates the benefits of using a multispecialist team focusing on both psychiatric and medical issues.

An association of AD and ANAs with psychiatric states has previously been described (Graus et al. 2010; Najjar et al. 2012; Kayser and Dalmau 2011). Likewise, immunological phenotypes within both humoral and cellular immunity arms have been reported to be abnormal in individuals with psychiatric disease, and specific serum biological biomarkers have been determined for major depressive disorders (Zandi et al. 2011; Najjar et al. 2013). Furthermore, inflammatory markers such as CRP, IL-6, and TNF- $\alpha$ have been associated with development or severity of psychiatric disorders such as depression, psychosis, and bipolar disorder (Najjar et al. 2013; Goldstein et al. 2015; Khandaker et al. 2018a, 2018b).

We did not record any clear elevations in CRP, but we did not use the high-sensitivity CRP assay, which might be more accurate. In addition, we have only measured cytokine levels systematically in blood. For more complete results, these levels should be assessed in the CSF. In addition, as it is known that immune cell population activity varies according to circadian rhythms, it will be important in the future to include a defined time of the day for sampling of blood in case this contributed to some of the variation.

The fact that $37 \%$ of the sample had complement activation, $17 \%$ had a positive ANA value, $11 \%$ had anti-TPO antibodies, and $20 \%$ had immunoglobulin abnormalities, taken together with frequent indication of $\mathrm{AD}$ in our cohort, is suggestive of immune dysregulation in these individuals. Whether there is a causal relationship between these immunological markers and disease prognosis is currently unclear, but their value as biomarkers of PANS warrants further investigation.

The cohort needs to be expanded and recruitment of suitable control groups would be required (e.g., a regular OCD sample without suspected PANS and healthy age-matched controls) to establish whether these potentially interesting findings have clinical utility. However, we do know that the complement system is a key player in autoimmunity and activation rarely seen in healthy individuals (McGeer et al. 2017). In addition, anti-TPO antibodies 
are described to be prevalent in only $1 \%-2 \%$ healthy children (Harel et al. 2006; Kroon et al. 2013). Interpretation of ANAs is more difficult as the ANA test has a high false positive rate, meaning that many children who do not have an AD can have elevated ANA levels. Studies have indicated that up to $15 \%$ of healthy children may have a positive ANA test and that it may be frequent in children with nonautoimmune inflammatory diseases, including both acute and chronic infections (Wananukul et al., 2005; Malleson et al. 2010).

Previous epidemiological studies have suggested a familial link between $\mathrm{AD}$ and $\mathrm{OCD}$ and tic disorders, but it remains unclear if this link is stronger in patients also fulfilling criteria for PANS (Pérez-Vigil et al., 2016; Mataix-Cols et al. 2018). However, dividing the total cohort into an autoimmune group consisting of diagnosed $\mathrm{AD}$ in the patient or a first-degree relative and a nonautoimmune group consisting of no diagnosed AD in either the patient or a first-degree relative did not generate any significant differences. The high prevalence of AD or inflammatory disorders in the patients and their relatives has also been reported from the Stanford cohort (Frankovich et al., 2015) and may suggest a genetic vulnerability in families with $\mathrm{AD}$ to present with the constellation of symptoms described as PANS. However, while the presence an$\mathrm{d} /$ or family history of AD may be a common feature in this patient group, more research is needed before this knowledge can guide diagnosis or treatment. The absence of AD in childhood does not necessarily mean that a person cannot fulfill the criteria for PANS as the onset of most ADs tends to occur later in life. Long-term followup of these patients using the Swedish health care registers will shed more light on the possible development of $\mathrm{AD}$ later in life.

It is important to note that many of the patients referred to our team with a suspected PANS-like presentation did not fulfill the regular PANS criteria or more stringent criteria that we used for inclusion in our cohort requiring the presence of somatic signs. The main reasons for exclusion from our cohort were absence of core symptoms such as OCD and/or anorexia, insidious symptom onset, and spontaneous resolution of symptoms before the assessment took place. However, many of these patients clearly exhibited an atypical onset of severe psychiatric symptoms, sometimes related to an infection or to an $\mathrm{AD}$. While we did not include these patients in our cohort, they were clearly disabled by their symptoms. Whether the PANS criteria are too narrow and need to be broadened to include psychiatric manifestations other than OCD/anorexia triggered by autoimmune or inflammatory disorders is an important area of enquiry.

There is a great deal of misinformation among the general public and professionals alike regarding the nature of PANS, which may lead to inadequate or unnecessary treatments (Gilbert et al. 2018). During the course of our study, it became more apparent that patients were often treated with long-term antibiotics and/or NSAIDs and, in a few patients, even IVIG, without having had a systematic assessment of their suspected PANS symptoms. For this reason, our team started to routinely taper down current medications aimed at treating the suspected PANS symptoms when the patient first presented at our clinic. Fortunately, referrals have become progressively more accurate and most of the patients currently referred to our team do meet the criteria for PANS. These results highlight the importance of educating colleagues and the public about PANS since they appear to represent a small fraction of all cases referred to specialist OCD and related disorder clinics such as ours. During the study period, more than 1500 patients were referred to our specialist OCD and related disorders clinic and only 47 (or $~ 3 \%$ ) met criteria for PANS.

The main strength of the study is the careful protocol-driven approach of data collection and the multidisciplinary nature of our team. However, the study was conducted in a naturalistic clinical setting, which means that some data points differed across patients. For example, more chronic or severe patients had blood tests taken more regularly. For those patients who had tests taken on different occasions, we tried to capture an active disease state, but this was not easy to establish using objective criteria. For instance, some patients had positive laboratory results when they were in a less severe phase of their illness, and these results are therefore not considered. Serologies and cultures were only taken when considered clinically motivated, which may be considered as a limitation. Antistreptococcal antibody titers were not part of the protocol since they may be elevated in many preschool and school children who are frequently exposed to streptococci in everyday life and their validity is therefore disputed (Delice et al. 2015). In an attempt to select a strictly defined cohort and minimize risk of misdiagnosis, we did not include patients who did not exhibit any kind of somatic symptom or sign. Somatic signs are not part of main PANS criteria, but are listed as secondary diagnostic criteria. Therefore, our cohort may differ from other cohorts that did not have such a requirement. Additional limitations include parentreported data on family history; the modest sample size, resulting in limited power for subgroup analyses or analyses of clinical trajectories; and relatively short follow-up time. However, we plan to follow these patients long term. As mentioned above, we cannot be sure if any of the potential biomarkers identified in this study are clinically useful until we compare results with those of a regular OCD and related disorders cohort. This work is currently ongoing.

\section{Conclusions}

We have successfully established a multidisciplinary PANS team and an assessment protocol. There was a strong indication of an association with AD in our cohort. Further work is needed to establish whether any of the potential biomarkers identified will be clinically useful. Long-term follow-up of these patients using the Swedish national registers will enable a deeper understanding of the course of this patient group.

\section{Clinical Significance}

A thorough multidisciplinary assessment is needed to confirm that patients fulfill strict criteria for PANS or PANS-like presentations, rather than regular OCD or other psychiatric disorders, as this will determine whether antibiotic, anti-inflammatory, or immunomodulatory treatments should be considered. The high rates of autoimmunity in this patient group and their families warrant close collaboration between psychiatric and pediatric services.

\section{Acknowledgments}

The authors thank the children and parents who participated in the research as well as the psychologists and nurses involved in the PANS team.

\section{Disclosures}

No competing financial interests exist.

\section{Supplementary Material}

Supplementary Table S1

Supplementary Table S2

Supplementary Table S3

Supplementary Table S4

Supplementary Table S5 


\section{References}

American Psychiatric Association: Diagnostic and Statistical Manual of Mental Disorders: DSM-5, 5th ed. Washington, DC: American Psychiatric Publishing; 2013.

Bäck SE, Nilsson JE, Fex G, Jeppson JO, Rosen U, Tryding N, von Schenck H, Norlund L: Towards common reference intervals in clinical chemistry: An attempt at harmonization between three hospital laboratories in Skåne, Sweden. Clin Chem Lab Med 37: 573-592, 1999.

Brimberg L, Benhar I, Mascaro-Blanco A, Alvarez K, Lotan D, Winter C, Klein J, Moses AE, Somnier FE, Leckman JF, Swedo SE, Cunningham MW, Joel D: Behavioral, pharmacological, and immunological abnormalities after streptococcal exposure: A novel rat model of Sydenham chorea and related neuropsychiatric disorders. Neuropsychopharmacology 37:2076-2087, 2012.

Brown K, Farmer C, Farhadian B, Hernandez J, Thienemann M, Frankovich J: Pediatric acute-onset neuropsychiatric syndrome response to oral corticosteroid bursts: An observational study of patients in an academic community-based PANS clinic. J Child Adolesc Psychopharmacol 27:629-639, 2017a.

Brown KD, Farmer C, Freeman GM Jr, Spartz EJ, Farhadian B, Thienemann M, Frankovich J: Effect of early and prophylactic nonsteroidal anti-inflammatory drugs on flare duration in pediatric acute-onset neuropsychiatric syndrome: an observational study of patients followed by an academic community-based pediatric acute-onset neuropsychiatric syndrome clinic. Child Adolesc Psychopharmacol 27:619-628, 2017b.

Busner J, Targum SD: The clinical global impressions scale: Applying a research tool in clinical practice. Psychiatry 4:28-32, 2007.

Chang K, Frankovich J, Cooperstock M, Cunningham MW, Latimer ME, Murphy TK, Pasternack M, Thienemann M, Williams K, Walter J, Swedo SE: Clinical evaluation of youth with pediatric acute-onset neuropsychiatric syndrome (PANS): Recommendations from the 2013 PANS Consensus Conference. J Child Adolesc Psychopharmacol 25:3-13, 2015.

Cooperstock MS, Swedo SE, Pasternack MS, Murphy TK: Clinical management of pediatric acute-onset neuropsychiatric syndrome: Part III-Treatment and prevention of infections. J Child Adolesc Psychopharmacol 27:594-606, 2017.

Cutforth T, DeMille MM, Agalliu I, Agalliu D: CNS autoimmune disease after Streptococcus pyogenes infections: Animal models, cellular mechanisms and genetic factors. Future Neurol 11:6376, 2016.

Dale RC, Brilot F: Autoimmune basal ganglia disorders. J Child Neurol 27:1470-1481, 2012.

Dalmau J, Gleichmann AJ, Hughes EJ, Rossi JE, Peng X, Lai M, Dessain SK, Rosenfeld MR, Balice-Gordon R, Lynch DR: AntiNMDA-receptor encephalitis: case series and analysis of the effects of antibodies. Lancet Neurol 7:1091-1098, 2008.

Delice S, Adaleti R, Sevan S, Alagoz P, Bedel A, Nuhoglu C, Aksaray S: Detection of upper limit of normal values of anti-DNase B antibody in children's age groups who were admitted to hospital with noninfectious reasons. North Clin Istanb 2:136-141, 2015.

Farhood Z, Ong AA, Discolo CM: PANDAS: A systematic review of treatment options. Int J Pediatr Otorhinolaryngol 89:149-153, 2016.

Frankovich J, Thienemann M, Pearlstein J, Crable A, Brown K, Chang K: Multidisciplinary clinic dedicated to treating youth with pediatric acute-onset neuropsychiatric syndrome: Presenting characteristics of the First 47 consecutive patients. J Child Adolesc Psychopharmacol 25:38-47, 2015.

Frankovich J, Swedo S, Murphy T, Dale RC, Agalliu D, Williams K, Daines M, Hornig M, Chugani H, Sanger T, Muscal E, Pasternack M, Cooperstock M, Gans H, Zhang Y, Cunningham M, Bernstein
G, Bromberg R, Willett T, Brown K, Farhadian B, Chang K, Geller D, Hernandez J, Sherr J, Shaw R, Latimer E, Leckman J, thienemann M: Clinical management of pediatric acute-onset neuropsychiatric syndrome: Part II-Use of Immunomodulatory therapies. J Child Adolesc Psychopharmacol 27:574-593, 2017.

Gilbert DL, Mink JW, Singer HS: A pediatric neurology perspective on pediatric autoimmune neuropsychiatric disorder associated with streptococcal infection and pediatric acute- onset neuropsychiatric syndrome. J Pediatr 199:243-251, 2018.

Goldstein BI, Lotrich F, Axelson DA, Gill MK, Hower H, Goldstein TR, Fan J, Yen S, Diler R, Dickstein D, Strober MA, Iyengar S, Ryan ND, Keller MB, Birmaher MB: Inflammatory markers among adolescents and young adults with bipolar spectrum disorders. J Clin Psychiatry 76:1556-1563, 2015.

Goodman WK, Price LH, Rasmussen SA, Mazure C, Fleischmann RL, Hill CL, Heninger GR, Charney DS: The Yale-Brown ObsessiveCompulsive Scale. I. Development, use, and reliability. Arch Gen Psychiatry 46:1006-1011, 1989.

Graus F, Saiz A, Dalmau J: Antibodies and neuronal autoimmune disorders of the CNS. J Neurol 257:509-517, 2010.

Hacohen Y, Wright S, Waters P, Agrawal S, Carr L, Cross H, De Sousa C, Devile C, Fallon P, Gupta R, Hedderly T, Hughes E, Kerr T, Lascelles K, Lin JP, Philip S, Pohl K, Prabahkar T, Smith M, Williams R, Clarke A, Hemingway C, Wassmer E, Vincent A, Lim MJ: Paediatric autoimmune encephalopathies: Clinical features, laboratory investigations and outcomes in patients with or without antibodies to known central nervous system autoantigens. J Neurol Neurosurg Psychiatry 84:748-755, 2013.

Harel L, Prais D, Uziel Y, Mukamel M, Hashkes P, Harel G, Amir J, Monselise Y, Press J: Increased prevalence of antithyroid antibodies and subclinical hypothyroidism in children with juvenile idiopathic arthritis. J Rheumatol 33:164-166, 2006.

Hesselmark E, Bejerot S: Biomarkers for diagnosis of pediatric acute neuropsychiatric syndrome (PANS)-Sensitivity and specificity of the Cunningham Panel. J Neuroimmunol 312:31-37, 2017a.

Hesselmark E, Bejerot S: Corrigendum to "Biomarkers for diagnosis of Pediatric Acute Neuropsychiatric Syndrome (PANS)-Sensitivity and specificity of the Cunningham Panel." J Neuroimmunol 313: 116-117, 2017b.

Kayser MS, Dalmau J: The emerging link between autoimmune disorders and neuropsychiatric disease. J Neuropsychiatry Clin Neurosci 23:90-97, 2011.

Khandaker GM, Stochl J, Zammit S, Goodyer I, Lewis G, Jones PB: Childhood inflammatory markers and intelligence as predictors of subsequent persistent depressive symptoms: A longitudinal cohort study. Psychol Med 48:1514-1522, 2018 a.

Khandaker GM, Zammit S, Burgess S, Lewis G, Jones PB: Association between a functional interleukin 6 receptor genetic variant and risk of depression and psychosis in a population-based birth cohort. Brain Behav Immun 69:264-272, 2018b.

Kroon MW, Vrijman C, Chandeck C, Wind BS, Wolkerstorfer A, Luiten RM, Bos JD, Geskus RB, van Trotsenburg P, Wietze van der Veen JP: High prevalence of autoimmune thyroiditis in children and adolescents with vitiligo. Horm Res Paediatr 79:137-144, 2013.

Kurlan R, Johnson D, Kaplan EL: Streptococcal infection and exacerbations of childhood tics and obsessive-compulsive symptoms: a prospective blinded cohort study. Pediatrics 121:1188-1197, 2008.

Latimer ME, L'Etoile N, Seidlitz J, Swedo SE: Therapeutic plasma apheresis as a treatment for 35 severely ill children and adolescents with pediatric autoimmune neuropsychiatric disorders associated with streptococcal infections. J Child Adolesc Psychopharmacol 25:70-75, 2015 .

Leckman JF, King RA, Gilbert DL, Coffey BJ, Singer HS, Dure LS 4th, Grantz H, Katsovich L, Lin H, Lombroso PJ, Kawikowa I, 
Johnson DR, Kurlan MR, Kaplan EL: Streptococcal upper respiratory tract infections and exacerbations of tic and obsessivecompulsive symptoms: A prospective longitudinal study. J Am Acad Child Adolesc Psychiatry, 50:108-118, 2011.

Leckman JF, Riddle MA, Hardin MT, Ort SI, Swartz KL, Stevenson J, Cohen DJ: The Yale Global Tic Severity Scale: Initial testing of a clinician-rated scale of tic severity. J Am Acad Child Adolesc Psychiatry 28:566-573, 1989.

Mahony T, Sidell D, Gans H, Brown K, Farhadian B, Gustafson M, Sherr J, Thienemann M, Frankovich J: Improvement of psychiatric symptoms in youth following resolution of sinusitis. Int $\mathrm{J}$ Pediatr Otorhinolaryngol 92:38-44, 2017a.

Mahony T, Sidell D, Gans H, Cooperstock M, Brown K, Cheung JM, Farhadian B, Gustafson M, Thienemann M, Frankovich J: Palatal petechiae in the absence of group a streptococcus in pediatric patients with acute-onset neuropsychiatric deterioration: A cohort study. J Child Adolesc Psychopharmacol 27:660-666, $2017 \mathrm{~b}$.

Malleson PN, Mackinnon MJ, Sailer-Hoeck M, Spencer CH: Review for the generalist: The antinuclear antibody test in children-when use it and what to do with a positive titer. Pediatr Rheumatol Online J 8:27, 2010.

Mataix-Cols D, Frans E, Pérez-Vigil A, Kuja-Halkola R, Gromark C, Isomura K, Fernández de la Cruz L, Serlachius E, Leckman JF, Crowley JJ, Rück C, Almqvist C, Lictenstein P, Larsson H: A totalpopulation multigenerational family clustering study of autoimmune diseases in obsessive-compulsive disorder and Tourette's/ chronic tic disorders. Mol Psychiatry 23:1652-1658, 2018.

McGeer PL, Lee M, McGeer EG: A review of human diseases caused or exacerbated by aberrant complement activation. Neurobiol Aging 52:12-22, 2017.

McGuire JF, Piacentini J, Storch EA, Murphy TK, Ricketts EJ, Woods DW, Walkup JW, Peterson AL, Wilhelm S, Lewin AB, McCracken JT, Leckman JF, Scahill L: A multicenter examination and strategic revisions of the Yale Global Tic Severity Scale. Neurology 90: 1711-1719, 2018.

Najjar S, Pearlman DM, Alper K, Najjar A, Devinsky O: Neuroinflammation and psychiatric illness. J Neuroinflammation 10:43, 2013.

Najjar S, Pearlman DM, Zagzag D, Golfinos J, Devinsky O: Glutamic acid decarboxylase autoantibody syndrome presenting as schizophrenia. Neurologist 18:88-91, 2012.

Parkin PC, Hamid J, Borkhoff CM, Abdullah K, Atenafu EG, Birken CS, Maquire JL, Azad A, Higgins V, Adeli K: Laboratory reference intervals in the assessment of iron status in young children. BMJ Paediatr Open 1:e000074, 2017.

Pérez-Vigil A, Fernández de la Cruz L, Brander G, Isomura K, Gromark C, Mataix-Cols D: The link between autoimmune diseases and obsessive-compulsive and tic disorders: A systematic review. Neurosci Biobeh Rev 71:542-562, 2016.

Ramanathan S, Mohammad SS, Brilot F, Dale RC: Autoimmune encephalitis: recent updates and emerging challenges. J Clin Neurosci 21:722-730, 2014.

Roche Diagnostics $\mathrm{GmbH}$ : Reference Intervals for Children and Adults: Elecsys Thyroid Tests. www.roche.com, 2009.
Shaffer D, Gould MS, Brasic J, Ambrosini P, Fisher P, Bird H, Aluwahlia S: A children's global assessment scale (CGAS). Arch Gen Psychiatry 40:1228-1231, 1983.

Sigra S, Hesselmark E, Bejerot S: Treatment of PANDAS and PANS: A systematic review. Neurosci Biobehav Rev 86:51-65, 2018.

Spartz EJ, Freeman GM Jr, J Brown K, Farhadian B, Thienemann M, Frankovich J: Course of neuropsychiatric symptoms after introduction and removal of nonsteroidal anti-inflammatory drugs: A pediatric observational study. J Child Adolesc Psychopharmacol 27:652-659, 2017.

Storch EA, Rasmussen SA, Price LH, Larson MJ, Murphy TK, Goodman WK: Development and psychometric evaluation of the Yale-Brown Obsessive-Compulsive Scale-Second edition. Psychol Assessment 2:223-232, 2010.

Swedo SE, Leonard HL, Garvey M, Mittleman B, Allen AJ, Perlmutter S, Lougee L, Dow S, Zamkoff J, Dubbert BK: Pediatric autoimmune neuropsychiatric disorders associated with streptococcal infections: Clinical description of the first 50 cases. Am J Psychiatry 155:264-271, 1998.

Thienemann M, Murphy T, Leckman J, Shaw R, Williams K, Kappahan C, Frankovich J, Geller D, Bernstein G, Chang K, Elia J, Swedo S: Clinical management of pediatric acute-onset neuropsychiatric syndrome: Part I-Psychiatric and Behavioral Interventions. J Child Adolesc Psychopharmacol 27:566-573, 2017.

Wananukul S, Voramethkul W, Kaewopas Y, Hanvivatvong O: Prevalence of positive antinuclear antibodies in healthy children. Asian Pac J Allergy Immunol 23:153, 2005.

Williams KA, Swedo SE, Farmer CA, Grantz H, Grant PJ, D'Souza P, Hommer R, Katsovich L, King RA, Leckman JF: Randomized, controlled trial of intravenous immunoglobulin for pediatric autoimmune neuropsychiatric disorders associated with streptococcal infections. J Am Acad Child Adolesc Psychiatry 55:860-867, 2016.

World Health Organization: International Statistical Classification of Diseases and Related Health Problems-Tenth Revision. Geneva, Switzerland, Elsevier Limited, 2011.

Yamada T: Serum amyloid A (SAA): A concise review on biology, assay methods and clinical usefulness. Clin Chem Lab Med 4: 381-388, 1999.

Yesiltepe Mutlu G, Hatun S: Use of vitamin D in children and adults: Frequently asked questions. J Clin Res Pediatr Endocrinol 10:301306, 2018.

Zandi MS, Irani SR, Lang B, Waters P, Jones PB, McKenna P, Coles AJ, Vincent A, Lennox BR: Disease-relevant autoantibodies in first episode schizophrenia. J Neurol 258:686-688, 2011.

Address correspondence to:

Caroline Gromark, MD

Department of Clinical Neuroscience

Karolinska Institutet

Gävlegatan 22

Stockholm SE-113 30

Sweden

E-mail: caroline.gromark@ki.se 